\title{
Straight-Line Embeddings of Two Rooted Trees in the Plane
}

\author{
A. Kaneko ${ }^{1}$ and M. Kano ${ }^{2}$ \\ ${ }^{1}$ Department of Electronic Engineering, Kogakuin University, \\ Nishi-Shinjuku, Shinjuku-ku, Tokyo 163-8677, Japan \\ kaneko@ee.kogakuin.ac.jp \\ ${ }^{2}$ Department of Computer and Information Sciences, \\ Ibaraki University, Hitachi 316-8511, Japan \\ kano@cis.ibaraki.ac.jp
}

\begin{abstract}
We prove the following theorem: Let $T_{1}$ and $T_{2}$ be two disjoint rooted trees with roots $v_{1}$ and $v_{2}$, respectively, and let $P$ be a set of $\left|T_{1} \cup T_{2}\right|$ points in the plane in general position containing two specified points $p_{1}$ and $p_{2}$. Then the union $T_{1} \cup T_{2}$ can be straight-line embedded onto $P$ such that $v_{1}$ and $v_{2}$ correspond to $p_{1}$ and $p_{2}$, respectively. Moreover, we give a $O\left(n^{2} \log n\right)$ time algorithm for finding such an embedding, where $n$ is the number of vertices contained in $T_{1} \cup T_{2}$.
\end{abstract}

\section{Introduction}

We consider finite planar graphs without loops or multiple edges. Let $G$ be a planar graph with vertex set $V(G)$ and edge set $E(G)$. We denote by $|G|$ the order of $G$, that is, $|G|=|V(G)|$. Given a planar graph $G$, let $P$ be a set of $|G|$ points in the plane (twodimensional Euclidean space) in general position (i.e., no three of them are collinear). Then $G$ is said to be line embedded onto $P$ or stright-line embedded onto $P$ if $G$ can be embedded in the plane so that every vertex of $G$ corresponds to a point of $P$, every edge corresponds to a straight-line segment, and no two straight-line segments intersect except their common endpoint. Namely, $G$ is line embedded onto $P$ if there exists a bijection $\varphi: V(G) \rightarrow P$ such that two points $\varphi(x)$ and $\varphi(y)$ are joined by a straight-line segment if and only if $x$ and $y$ are joined by an edge of $G$ and all two distinct open straight-line segments have no point in common. We call such a bijection a line embedding or a straight-line embedding of $G$ onto $P$.

In this paper we consider a line embedding having one more property. Let $G$ be a planar graph with $n$ specified vertices $v_{1}, v_{2}, \ldots, v_{n}$, and let $P$ be a set of $|G|$ points in the plane in general position containing $n$ specified points $p_{1}, p_{2}, \ldots, p_{n}$. Then we say that $G$ is strongly line embedded onto $P$ if $G$ can be line embedded onto $P$ so that, 

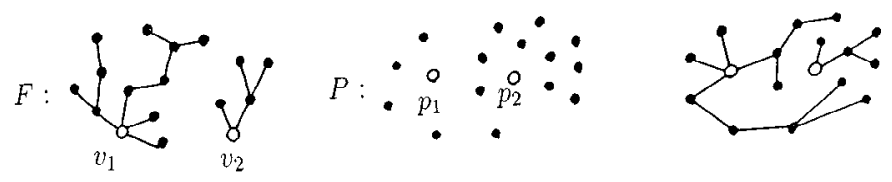

Fig. 1. A rooted forest $F$ and its strong line embedding onto $P$.

for every $1 \leq i \leq n, v_{i}$ corrresponds to $p_{i}$, that is, if there exists a line embedding $\varphi: V(G) \rightarrow P$ such that $\varphi\left(v_{i}\right)=p_{i}$ for all $1 \leq i \leq n$. The line embedding mentioned above is called a strong line embedding of $G$ onto $P$. A tree with one specified vertex $v$ is usually called a rooted tree with root $v$. Given $n$ disjoint rooted trees $T_{i}$ with root $v_{i}$, $1 \leq i \leq n$, the union $T_{1} \cup T_{2} \cup \cdots \cup T_{n}$, whose vertex set is $V\left(T_{1}\right) \cup V\left(T_{2}\right) \cup \cdots \cup V\left(T_{n}\right)$ and whose edge set is $E\left(T_{1}\right) \cup E\left(T_{2}\right) \cup \cdots \cup E\left(T_{n}\right)$, is called a rooted forest with roots $v_{1}, v_{2}, \ldots, v_{n}$, which are specified vertices of it.

We begin with the following theorem, which was conjectured by Perles [5] and partially solved by Pach and Törőcsik [4]; a simpler proof can be found in [7]. Another related result can be found in [2].

Theorem A [3]. A rooted tree $T$ can be strongly line embedded onto every set of $|T|$ points in the plane in general position containing a specified point.

In this paper we prove the following theorem.

Theorem 1. A rooted forest $F$ consisting of two rooted trees can be strongly line embedded onto every set of $|F|$ points in the plane in general position containing two specified points (see Fig. 1).

Moreover, our proof of the theorem gives an $O\left(|F|^{2} \log |F|\right)$ time algorithm for finding a strong line embedding. Before giving a proof, we mention that there exist rooted forests consisting of four rooted trees which cannot be strongly line embedded onto certain sets of points in the plane in general position containing four specified points. An example of such a forest and a set of points are given in Fig. 2. However, we propose the following conjecture and problem.

Conjecture B. A rooted forest $F$ consisting of three rooted trees can be strongly line embedded onto every set of $|F|$ points in the plane in general position containing three specified points.
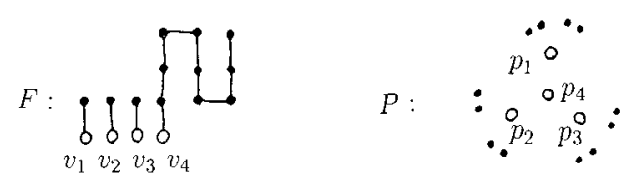

Fig. 2. A rooted forest $F$ which cannot be strongly line embedded onto $P$. 
Problem C. Let $F:=T_{1} \cup T_{2} \cup \cdots \cup T_{n}$ be a rooted forest with roots $v_{1}, v_{2}, \ldots, v_{n}$ and let $P$ be a set of $|F|$ points in the plane in general position containing $n$ specified points $p_{1}, p_{2}, \ldots, p_{n}$. Find a sufficient condition for $F$ to be strongly line embedded onto $P$.

\section{Proof of Theorem}

In order to prove our theorem, we need some notation and definitions. Let $X$ be a set of points in the plane. We denote by $\operatorname{conv}(X)$ the convex hull of $X$, which is the smallest convex set containing $X$. A point of $\operatorname{conv}(X)$ not lying on the boundary of $\operatorname{conv}(X)$ is called an interior point of $\operatorname{conv}(X)$. It is obvious that if $X$ consists of points in general position, then every point of $X$ lying on the boundary of $\operatorname{conv}(X)$ is a vertex of $\operatorname{conv}(X)$. For two points $x$ and $y$ in the plane, we denote by $\overline{x y}$ the straight-line segment joining $x$ to $y$.

Let $G$ be a graph. For a vertex $v$ of $G$, we denote by $\operatorname{deg}_{G}(v)$ the degree of $v$ in $G$. For a subset $S \subseteq V(G)$, we denote by $G-S$ the graph obtained from $G$ by deleting the vertices in $S$ together with their incident edges, and if $S=\{v\}$, then we write $G-v$ for $G-\{v\}$. Furthermore, the subgraph of $G$ induced by $S$ is denoted by $\langle S\rangle_{G}$, which is equal to $G-(V(G) \backslash S)$.

Let $P$ be a set of points in the plane in general position containing specified points. For convenience, we call a nonspecified point of $P$ an ordinary point, and denote the set of ordinary points of $P$ by $O(P)$. For a region $R$ in the plane, we state which points of $P$ lying on the boundary of $R$ are contained in $P \cap R$ one by one. Thus when we introduce a new notation on a region, we do not mention its boundary.

For three noncollinear points $x, y$, and $p$ in the plane, the plane is partitioned into two regions by two rays emanating from $p$ and passing through $x$ and $y$, respectively. We denote by Rgn(xpy) the region whose induced angle is less than $\pi$, that is, $\operatorname{Rgn}(x p y)$ denotes the internal region. Similarly, for noncollinear point $x$ and ray $r$ from $p$, and for noncollinear rays $r_{1}$ and $r_{2}$ from $p, \operatorname{Rgn}(x p r)$ and $\operatorname{Rgn}\left(r_{1} p r_{2}\right)$ denote the similar internal regions (see Fig. 3). Moreover, for two rays $r_{1}$ and $r_{2}$ emanating from $x_{1}$ and $x_{2}$, respectively, we define the region $\operatorname{Rgn}\left(r_{1} x_{1} x_{2} r_{2}\right)$ as Fig. 3. If we consider a region including all its boundary, then we call it a closed region, and if we consider a region without its boundary, then we call it an open region.

Before giving some lemmas, we explain a brief sketch of the proof of Theorem 1 so that readers can guess the reason why the following lemmas are given. We first deal with
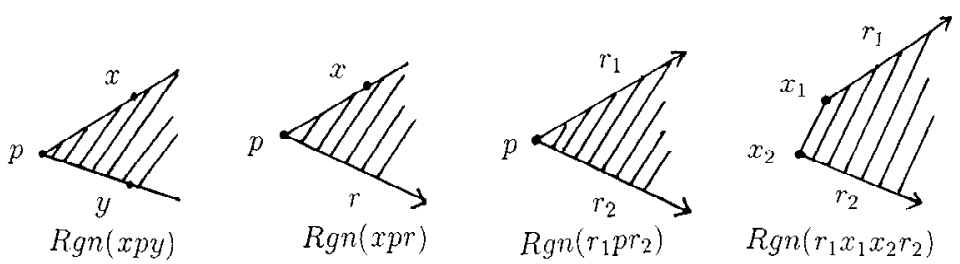

Fig. 3. Regions $\operatorname{Rgn}(x p y), \operatorname{Rgn}(x p r), \operatorname{Rgn}\left(r_{1} p r_{2}\right)$, and $\operatorname{Rgn}\left(r_{1} x_{1} x_{2} r_{2}\right)$. 
the case where one of the specified points lies on the boundary of $\operatorname{conv}(P)$, and next consider the case where both specified points are interior points of $\operatorname{conv}(P)$. In order to prove the latter case, we partition the bigger rooted tree into some rooted subtrees, which have some new common roots, and also partiton the point set $P$ into some subsets, which have some common new specified points. Then we try to embed two or more rooted subtrees onto the corresponding subset $Q$ of $P$ under the condition that some of the specified points of $Q$ lie on the boundary of $\operatorname{conv}(Q)$.

Lemma 2. A tree $T$ has a vertex $v$ such that every component of $T-v$ has order less than or equal to $|T| / 2$.

Proof. Choose a vetex $v$ of $T$ so that the maximum order of the components of $T-v$ is minimum among all vertices of $T$. Then $v$ satisfies the condition of this lemma.

Note that if, for a vertex $x$ of a tree $T, T-x$ has a component $C$ whose order is greater than $|T| / 2$, then the vertex $y$ of $C$ adjacent to $x$ has the property that every component of $T-y$ has order less than $|C|$. We can find the desired vertex $v$ in the above lemma in $O(|T| \log |T|)$ time by making use of this fact.

Lemma 3. Let $T$ be a tree with two specified vertices $v_{1}$ and $v_{2}$, and let $P$ be a set of $|T|$ points in the plane in general position containing two specified points $p_{1}$ and $p_{2}$. If $p_{1}$ and $p_{2}$ are consecutive vertices of $\operatorname{conv}(P)$, then $T$ can be strongly line embedded onto $P$.

Proof. We prove the lemma by induction on $|T|$. By a suitable rotation of the plane and by the symmetry of $p_{1}$ and $p_{2}$, we may assume that $p_{1}$ lies on the bottom of $\operatorname{conv}(P)$ and that $p_{2}$ lies to the right of $p_{1}$. Suppose first $\operatorname{deg}_{T}\left(v_{1}\right)=1$. In this case we take a vertex $q$ of $\operatorname{conv}\left(P \backslash\left\{p_{1}\right\}\right)$ such that the straight-line segment $\overline{q p_{2}}$ is an edge of $\operatorname{conv}\left(P \backslash\left\{p_{1}\right\}\right)$ and $\overline{p_{1} q}$ intersects $\operatorname{conv}\left(P \backslash\left\{p_{1}\right\}\right)$ at only $q$. Let $u$ be the vertex of $T$ adjacent to $v_{1}$. Then, by induction, the tree $T-v_{1}$ with two specified vertices $u$ and $v_{2}$ is strongly line embedded onto $P \backslash\left\{p_{1}\right\}$ with specified points $q$ and $p_{2}$. By adding $\overline{p_{1} q}$ to this embedding, we can get the desired strong line embedding of $T$.

We next assume $\operatorname{deg}_{T}\left(v_{1}\right) \geq 2$. Let $D$ be a component of $T-v_{1}$ not containing $v_{2}$. Then $1 \leq|D| \leq|T|-2=|P|-2$, and so there exists a line $l$ passing through $p_{1}$ such that the number of ordinary points of $P$ lying on or to the left of $l$ is equal to $|D|$. We denote the set of these ordinary points of $P$ by $Q$. Then, by Theorem A, the rooted tree $\left\langle D \cup\left\{v_{1}\right\}\right\rangle_{T}$ with root $v_{1}$ is strongly line embedded onto $Q \cup\left\{p_{1}\right\}$ with specified point $p_{1}$. Furthermore, it follows from the inductive hypothesis that $T-V(D)$ with specified vertices $v_{1}$ and $v_{2}$ is strongly line embedded onto $P \backslash Q$ with specified points $p_{1}$ and $p_{2}$. By combining the above two embeddings, we can obtain the desired strong line embedding of $T$ onto $P$.

Lemma 4. Let $T_{1} \cup T_{2}$ be a rooted forest with roots $v_{1}$ and $v_{2}$, and let $P$ be a set of $\left|T_{1} \cup T_{2}\right|$ points in the plane in general position containing two specified points $p_{1}$ and $p_{2}$. If $p_{1}$ is a vertex of $\operatorname{conv}(P)$, then $T_{1} \cup T_{2}$ can be strongly line embedded onto $P$. 
Proof. We prove the lemma by induction on $\left|T_{1} \cup T_{2}\right|$. Suppose first $\operatorname{deg}_{T_{1}}\left(v_{1}\right)=1$. We take a vertex $q$ of $\operatorname{conv}\left(P \backslash\left\{p_{1}\right\}\right)$ such that $q \neq p_{2}$ and $\overline{p_{1} q}$ intersects $\operatorname{conv}\left(P \backslash\left\{p_{1}\right\}\right)$ at only $q$. Let $u$ be the vertex of $T_{1}$ adjacent to $v_{1}$. Then, by induction, the rooted forest $\left(T_{1}-v_{1}\right) \cup T_{2}$ with roots $u$ and $v_{2}$ can be strongly line embedded onto $P \backslash\left\{p_{1}\right\}$ with specified points $q$ and $p_{2}$. By adding $\overline{p_{1} q}$ to this embedding, we get the desired embedding of $T_{1} \cup T_{2}$ onto $P$.

We next assume $\operatorname{deg}_{T_{1}}\left(v_{1}\right) \geq 2$. Let $D$ be one of the smallest components of $T_{1}-v_{1}$. Then $|D| \leq\left(\left|T_{1}\right|-1\right) / 2 \leq(|P|-2) / 2$, and so at least one of the two open regions detemined by the line passing through $p_{1}$ and $p_{2}$ contains at least $|D|$ ordinary points of $P$. Thus there exists a line $l$ passing through $p_{1}$ such that one of the open regions determined by $l$, say $R$, contains exactly $|D|$ ordinary points of $P$ and does not contain $p_{2}$. Then, by Theorem A, $\left\langle D \cup\left\{p_{1}\right\}\right\rangle_{T_{1}}$ with root $v_{1}$ is strongly line embedded onto $(R \cap O(P)) \cup\left\{p_{1}\right\}$ with specified point $p_{1}$. By the inductive hypothesis, $\left(T_{1}-V(D)\right) \cup T_{2}$ with roots $v_{1}$ and $v_{2}$ is strongly line embedded onto $P \backslash(R \cap O(P))$ with specified points $p_{1}$ and $p_{2}$. Combining these embeddings, we can obtain the desired strong line embedding of $T_{1} \cup T_{2}$ onto $P$.

Lemma 5. Let $F:=T_{1} \cup T_{2} \cup T_{3}$ be a rooted forest with roots $v_{1}, v_{2}, v_{3}$, and let $P$ be a set of $|F|$ points in the plane in general position containing three specified points $p_{1}, p_{2}, p_{3}$. If $p_{1}$ and $p_{2}$ are consecutive vertices of $\operatorname{conv}(P)$, then $F$ can be strongly line embedded onto $P$.

Proof. Without loss of generality, we may assume that $p_{1}$ lies on the bottom of $\operatorname{conv}(P)$ and that $p_{2}$ lies to the right of $p_{1}$. We consider only rays $r$ emanating from $p_{1}$ and going upward, and so a ray means such a ray. Given a ray $r$, let $P(r)$ denote the set of points of $P$ lying on or to the left of $r$. Then $p_{1} \in P(r)$ for every ray $r$, and there exists a ray $r_{1}$ such that either (i) $\left|P\left(r_{1}\right)\right|=\left|T_{1}\right|$ and $P\left(r_{1}\right)$ does not contain $p_{3}$; or (ii) $\left|P\left(r_{1}\right)\right|=\left|T_{1}\right|+\left|T_{3}\right|$ and $P\left(r_{1}\right)$ contains $p_{3}$. If $r_{1}$ satisfies (i), then $T_{1}$ and $T_{2} \cup T_{3}$ are strongly line embedded onto $P\left(r_{1}\right)$ and onto $P \backslash P\left(r_{1}\right)$, respectively, by Theorem A and by Lemma 4. Similarly, if $r_{1}$ satisfies (ii), then $T_{1} \cup T_{3}$ and $T_{2}$ are strongly line embedded onto $P\left(r_{1}\right)$ and onto $P \backslash P\left(r_{1}\right)$, respectively. Therefore $T_{1} \cup T_{2} \cup T_{3}$ can be strongly line embedded onto $P$.

Proof of Theorem 1. Let $F:=T_{1} \cup T_{2}$ be a rooted forest with roots $v_{1}$ and $v_{2}$, and let $P$ be a set of $|F|$ points in the plane in general position containing two specified points $p_{1}$ and $p_{2}$. Then $O(P)=P \backslash\left\{p_{1}, p_{2}\right\}$, which is the set of ordinary points of $P$.

We may assume that $\left|T_{1}\right| \geq\left|T_{2}\right| \geq 2$ since if $\left|T_{2}\right|=1$, then the theorem follows from the fact that a strong line embedding of $T_{1}$ onto $P \backslash\left\{p_{2}\right\}$ is also a strong line embedding of $F$ onto $P$. Put $n_{1}:=\left|T_{1}\right|-1$ and $n_{2}:=\left|T_{2}\right|-1$, which are equal to the numbers of ordinary points of $P$ added to $p_{1}$ and to $p_{2}$ to construct $T_{1}$ and $T_{2}$, respectively.

We now prove the theorem. By Lemma 4 , we may assume that both $p_{1}$ and $p_{2}$ are interior points of $\operatorname{conv}(P)$. By a suitable rotation of the plane, we may assume that both $p_{1}$ and $p_{2}$ lie on the same horizontal line and that $p_{1}$ lies to the left of $p_{2}$. Moreover, since every line passing through $p_{1}$ or $p_{2}$ is an oriented line going upward, a line passing through $p_{1}$ or $p_{2}$ means an oriented line passing through $p_{1}$ or $p_{2}$ and going upward. A 
line passing neither $p_{1}$ nor $p_{2}$ is an oriented line or a usual unoriented line, and we state it one by one.

Claim 1. We may assume that for every line l passing through $p_{1}$, the number $f(l)$ of ordinary points of $P$ lying on or to the left of $l$ is less than $n_{1}$. In particular, we may assume that the number of ordinary points of $P$ lying above (below) the horizontal line passing through $p_{1}$ and $p_{2}$ is less than $n_{1}$.

Proof. Here we consider only lines passing through $p_{1}$, and so a line means such a line. Suppose that there exists a line $l_{1}$ such that $f\left(l_{1}\right) \geq n_{1}$. Since $\left(n_{1}+n_{2}\right) / 2 \leq n_{1}$, there exists a line $l_{2}$ with $f\left(l_{2}\right) \leq n_{1}$, which is obtained from the horizontal line passing through $p_{1}$ and $p_{2}$ by an infinitesimal clockwise or counterclockwise rotation around $p_{1}$. Since every line passes through at most one ordinary point of $P$, when we rotate a line from $l_{2}$ to $l_{1}$ around $p_{1}$, the value of $f$ changes \pm 1 according to whether the line hits or passes an ordinary point of $P$. Hence there exists a line $l_{3}$ such that $f\left(l_{3}\right)=n_{1}$. Then $T_{1}$ and $T_{2}$ are strongly line embedded onto the set of points of $P$ lying on or to the left of $l_{3}$ and onto the set of points of $P$ lying to the right of $l_{3}$, respectively. Therefore $F$ can be strongly line embedded onto $P$. Consequently, we may assume that $f(l)<n_{1}$ for every $l$.

Define an integer $M$ by

$$
M:=\max \{f(l)\},
$$

where the maximum is taken over all the lines $l$ passing through $p_{1}$ except the horizontal line passing through $p_{1}$ and $p_{2}$, and $f(l)$ is defined as in Claim 1. Then by Claim 1 we have

$$
\left(n_{1}+n_{2}\right) / 2 \leq M<n_{1} .
$$

Let $D_{1}, D_{2}, \ldots, D_{m}$ be the components of $T_{1}-v_{1}$ such that $\left|D_{1}\right| \geq\left|D_{2}\right| \geq \cdots \geq\left|D_{m}\right|$.

Claim 2. We may assume $\left|D_{1}\right|>M$.

Proof. Suppose that $\left|D_{1}\right| \leq M$. Here we consider only lines passing through $p_{1}$, and so a line means such a line. If there exists a line $l_{1}$ with $f\left(l_{1}\right) \leq\left|D_{1}\right|$, then there exists a line $l_{2}$ such that $f\left(l_{2}\right)=\left|D_{1}\right|$. Then, by Theorem A, the rooted tree $\left\langle D_{1} \cup\left\{v_{1}\right\}\right\rangle_{T_{1}}$ with root $v_{1}$ is strongly line embedded onto the set of points of $P$ lying on or to the left of $l_{2}$ with specified point $p_{1}$. By Lemma 4 , the rooted forest $\left(T_{1}-V\left(D_{1}\right)\right) \cup T_{2}$ with roots $v_{1}$ and $v_{2}$ is strongly line embedded onto the set of points of $P$ lying on or to the right of $l_{2}$ with specified points $p_{1}$ and $p_{2}$. Hence $F$ can be strongly line embedded onto $P$. Therefore we assume that, for every line $l$, we have $f(l)>\left|D_{1}\right|$.

We write $r_{0}$ for the ray emanating from $p_{1}$ and passing through $p_{2}$. Then since $f(l)>\left|D_{i}\right|$, for $1 \leq i \leq m-1$, we can inductively take a ray $r_{i}$ emanating from $p_{1}$ and not passing through any ordinary points of $P$ such that the open region $\operatorname{Rgn}\left(r_{i-1} p_{1} r_{i}\right)$ contains exactly $\left|D_{i}\right|$ ordinary points of $P$. Then each rooted tree $\left\langle D_{i} \cup\left\{v_{1}\right\}\right\rangle_{T_{1}}$ with root $v_{1}$ is strongly line embedded onto $\left(\operatorname{Rgn}\left(r_{i-1} p_{1} r_{i}\right) \cap O(P)\right) \cup\left\{p_{1}\right\}$ with specified point 
$p_{1}$. Moreover, by (1), the ray $r_{m}$ goes downward, and thus $T_{2}$ is strongly line embedded onto $\left(\operatorname{Rgn}\left(r_{m} p_{1} r_{0}\right) \cap O(P)\right) \cup\left\{p_{2}\right\}$. Therefore $F$ can be strongly line embedded onto $P$. Consequently we may assume that $\left|D_{1}\right|>M$.

By Lemma 2, $D_{1}$ has a vetex $w_{1}$ such that each component of $D_{1}-w_{1}$ has order less than or equal to $\left|D_{1}\right| / 2$. Let $A_{1}, A_{2}, \ldots, A_{t}$ be the components of $D_{1}-w_{1}$ such that $A_{1}$ is the component containing the vertex adjacent to $v_{1}$ in $T_{1}$ and $A_{2}$ is the largest component among $A_{2}, A_{3}, \ldots, A_{t}$. Note that $A_{1}=\emptyset$ if $v_{1}$ and $w_{1}$ are adjacent in $T_{1}$. Let $w_{2} \in A_{2}$ be the vertex adjacent to $w_{1}$. Define $B_{4}:=A_{2}$ and

$$
B_{2}:=A_{1} \cup\left(\bigcup_{i=3}^{r} A_{i}\right) \quad \text { if } t \geq 3, \quad \text { and otherwise } \quad B_{2}:=A_{1},
$$

where the integer $r, 3 \leq r \leq t$, is chosen as large as possible subject to $\left|B_{2}\right|<M$. In particular, if $r<t$, then $\left|B_{2} \cup A_{r+1}\right| \geq M$. Note that $\left|A_{1}\right|<M$ as $\left|A_{1}\right| \leq\left|D_{1}\right| / 2 \leq$ $\left(n_{1}-1\right) / 2 \leq\left(n_{1}+n_{2}\right) / 2-1 \leq M-1$. We define

$$
B_{3}:=A_{r+1} \cup \cdots \cup A_{t} \quad \text { if } \quad r<t, \quad \text { and otherwise } \quad B_{3}:=\emptyset .
$$

Moreover, put

$$
B_{1}:=D_{2} \cup \cdots \cup D_{m}=T_{1}-\left(V\left(D_{1}\right) \cup\left\{v_{1}\right\}\right) .
$$

Then $V\left(T_{1}\right)=V\left(B_{1}\right) \cup V\left(D_{1}\right) \cup\left\{v_{1}\right\}, V\left(D_{1}\right)=V\left(B_{2}\right) \cup V\left(B_{3}\right) \cup V\left(B_{4}\right) \cup\left\{w_{1}\right\}$, and $w_{2} \in B_{4}$ (see Fig. 4).

Claim 3. We may assume that, for every line l passing through $p_{1}$, the number $f(l)$ of ordinary points of $P$ lying on or to the left of $l$ is greater than or equal to $\left|B_{2}\right|+1$.

Proof. Suppose that there exists a line $l_{1}$ passing through $p_{1}$ for which $f\left(l_{1}\right) \leq\left|B_{2}\right|$. Then by the definition of $B_{2}$, we have $\left|B_{2}\right|+1 \leq M$, and thus we can find a line $l_{2}$ through $p_{1}$ such that $f\left(l_{2}\right)=\left|B_{2}\right|+1$ and $l_{2}$ passes through an ordinary point $q$ of $P$. Let $Q$ denote the set of ordinary points of $P$ lying on or to the left of $l_{2}$. Then, by Lemma 3, the rooted tree $\left\langle B_{2} \cup\left\{v_{1}, w_{1}\right\}\right\rangle_{T_{1}}$ with two specified vertices $v_{1}$ and $w_{1}$ is strongly line embedded onto $Q \cup\left\{p_{1}\right\}$ with two specified points $p_{1}$ and $q$. Similarly, by Lemma 5, the rooted forest $\left\langle B_{1} \cup\left\{v_{1}\right\}\right\rangle_{T_{1}} \cup\left\langle B_{3} \cup B_{4} \cup\left\{w_{1}\right\}\right\rangle_{T_{1}} \cup T_{2}$ with roots $v_{1}, w_{1}, v_{2}$ can be strongly line embedded onto $(O(P) \backslash Q) \cup\left\{p_{1}, q, p_{2}\right\}$ with specified points $p_{1}, q, p_{2}$. Therefore $T_{1} \cup T_{2}$ can be strongly line embedded onto $P$.

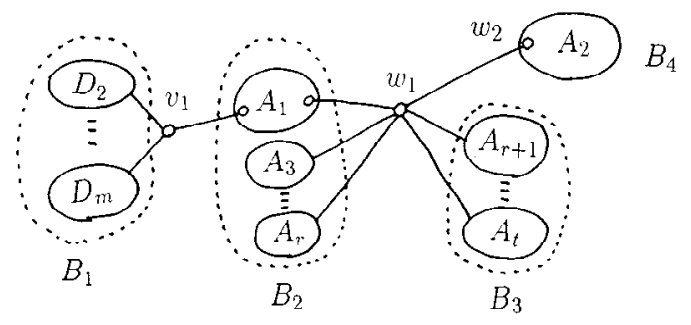

Fig. 4. The rooted tree $T_{1}$. 
Claim 4. We may assume $B_{3} \neq \emptyset$.

Proof. Suppose $B_{3}=\emptyset$. Let $l_{0}$ be the horizontal unoriented line passing through both $p_{1}$ and $p_{2}$, and let $r_{0}$ be the ray emanating from $p_{1}$ and passing through $p_{2}$.

Without loss of generality, we may assume that the number of ordinary points of $P$ lying above $l_{0}$ is greater than or equal to $\left(n_{1}+n_{2}\right) / 2$. Since $\left|B_{4}\right| \leq\left|D_{1}\right| / 2 \leq$ $\left(n_{1}+n_{2}\right) / 2-1$, there exists a ray $r_{1}$ emanating from $p_{1}$, going upward, and passing through an ordinary point $q$ of $P$ such that the closed region $\operatorname{Rgn}\left(r_{0} p_{1} r_{1}\right)$ contains exactly $\left|B_{4}\right|+1$ ordinary points of $P$.

By Claim 3, we can find a ray $r_{2}$ emanating from $p_{1}$ and not passing through any ordinary point of $P$ such that the closed region $\operatorname{Rgn}\left(r_{1} p_{1} r_{2}\right)$ contains $\left|B_{2}\right|+1$ ordinary points of $P$. Since $\left|B_{4}\right|+\left|B_{2}\right|+1=\left|D_{1}\right|>M$, and by the definition of $M, r_{2}$ must go downward, that is, $r_{2}$ lies below $l_{0}$. Then, by Thorem $A$, the rooted tree $\left\langle B_{4} \cup\left\{w_{1}\right\}\right\rangle_{T_{1}}$ with root $w_{1}$ is strongly line embedded onto $\operatorname{Rgn}\left(r_{0} p_{1} r_{1}\right) \cap O(P)$ with specified point $q$. By Lemma 3, the rooted tree $\left\langle B_{2} \cup\left\{w_{1}, v_{1}\right\}\right\rangle_{T_{1}}$ with two specified vertices $v_{1}$ and $w_{1}$ can be strongly line embedded onto $\left(\operatorname{Rg} n\left(r_{1} p_{1} r_{2}\right) \cap O(P)\right) \cup\left\{p_{1}\right\}$ with two specified points $p_{1}$ and $q$. Moreover, since $r_{2}$ goes downward, the two rooted trees $\left\langle B_{1} \cup\left\{v_{1}\right\}\right\rangle_{T_{1}}$ with root $v_{1}$ and $T_{2}$ with root $v_{2}$ can be strongly line embedded onto $\left(\operatorname{Rgn}\left(r_{2} p_{1} r_{0}\right) \cap O(P)\right) \cup\left\{p_{1}, p_{2}\right\}$ by Lemma 4 . Therefore $T_{1} \cup T_{2}$ is strongly line embedded onto $P$. Consequently we may assume $B_{3} \neq \varnothing$.

Let $l_{0}$ and $r_{0}$ denote the same line and ray as in the proof of Claim 4. Let $r_{0}^{*}$ be the ray from $p_{1}$ which lies on $l_{0}$ and whose direction is opposite to $r_{0}$. By Claim 3, we can take a ray $r_{3}$ emanating from $p_{1}$, passing through no ordinary point of $P$, and going upward such that the open region $\operatorname{Rgn}\left(r_{0}^{*} p_{1} r_{3}\right)$ contains $\left|B_{2}\right|$ ordinary points of $P$. Since $B_{3} \neq \emptyset$, we have $\left|B_{2} \cup B_{4}\right| \geq\left|B_{2} \cup A_{r+1}\right| \geq M$, which implies that the region above $l_{0}$ does not contain more than $\left|B_{2} \cup B_{4}\right|$ ordinary points of $P$. Thus the region below $l_{0}$ contains at least $n_{1}+n_{2}-\left|B_{2} \cup B_{4}\right|$ ordinary points of $P$. Since $n_{1}+n_{2}-\left|B_{2} \cup B_{4}\right| \geq\left|B_{3}\right|+2$, we can find a ray $r_{4}$ emanating from $p_{1}$, passing through no ordinary points of $P$, and going downward such that the open region $\operatorname{Rgn}\left(r_{0}^{*} p_{1} r_{4}\right)$ contains exactly $\left|B_{3}\right|+2$ ordinary points of $P$.

Let $l_{3}$ be the line containing $r_{3}$ and going upward, and let $r_{3}^{*}$ be the ray from $p_{1}$ opposite to $r_{3}$. Note that we may assume that $l_{3}$ passes through no ordinary point of $P$ because $r_{3}$ does not pass through any ordinary points. It follows from Claim 3 that $f\left(l_{3}\right) \geq\left|B_{2}\right|+1$, and so the open region $\operatorname{Rgn}\left(r_{0}^{*} p_{1} r_{3}^{*}\right)$ contains at least one ordinary point of $P$. Similarly, since $\left|B_{2}\right|+\left|B_{3}\right|+2>M$, the open region $\operatorname{Rgn}\left(r_{3}^{*} p_{1} r_{4}\right)$ contains at least one ordinary point of $P$. We choose two ordinary points $q_{1} \in \operatorname{Rgn}\left(r_{0}^{*} p_{1} r_{3}^{*}\right)$ and $q_{2} \in \operatorname{Rgn}\left(r_{3}^{*} p_{1} r_{4}\right)$ so that the intersection of the line segment $\overline{q_{1} q_{2}}$ with $r_{3}^{*}$ is closest to $p_{1}$ among all the pairs of ordinary points in $\operatorname{Rgn}\left(r_{0}^{*} p_{1} r_{3}^{*}\right)$ and those in $\operatorname{Rgn}\left(r_{3}^{*} p_{1} r_{4}\right)$ (see Fig. 5). In particular, the open triangle surrounded by $r_{0}^{*}, r_{4}$, and the line passing through $q_{1}$ and $q_{2}$ contains no ordinary point of $P$.

Let $l_{5}$ denote the unoriented line passing through $q_{1}$ and $q_{2}$. Let $r_{a}$ be a ray from $q_{1}$ passing through no ordinary point of $P$ except $q_{1}$ such that $r_{a}$ does not intersect $l_{3}$ and the open region $\operatorname{Rgn}\left(r_{a} q_{1} p_{1} r_{3}\right)$ contains exactly $\left|B_{2}\right|$ ordinary points, which is equal to the number of ordinary points in $\operatorname{Rgn}\left(r_{0}^{*} p_{1} r_{3}\right)$. Then since the open triangle surrounded by $r_{0}^{*}, r_{4}$, and $l_{5}$ contains no ordinary points of $P, r_{a}$ lies below $l_{5}$ and above the line 


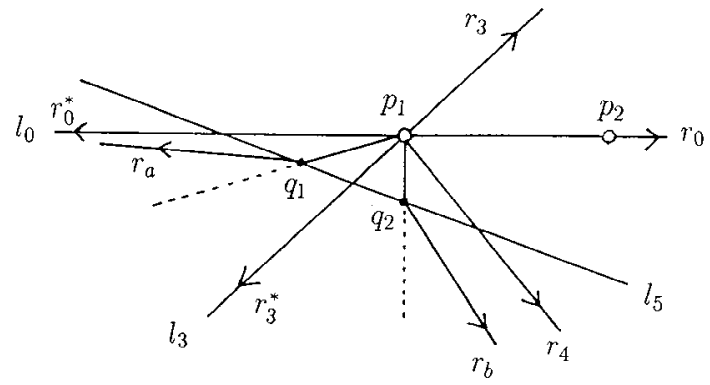

Fig. 5. $\operatorname{Rgn}\left(r_{a} q_{1} q_{2} r_{b}\right)$ does not contain $p_{2}$.

passing through $p_{1}$ and $q_{1}$. Similarly, let $r_{b}$ be a ray from $q_{2}$ passing through no ordinary point of $P$ except $q_{2}$ such that $r_{b}$ does not intersect $l_{3}$ and the open region $\operatorname{Rgn}\left(r_{a} q_{1} q_{2} r_{b}\right)$ contains exactly $\left|B_{3}\right|$ ordinary points of $P$ (see Fig. 5). Then $r_{b}$ lies below $l_{5}$ and to the right of the line passing through $p_{1}$ and $q_{2}$. Moreover, note that $q_{1} \notin \operatorname{Rgn}\left(r_{a} q_{1} p_{1} r_{3}\right)$ and $q_{1}, q_{2} \notin \operatorname{Rgn}\left(r_{a} q_{1} q_{2} r_{b}\right)$. By the properties of $r_{a}$ and $r_{b}$, the open regions $\operatorname{Rgn}\left(r_{a} q_{1} p_{1} r_{3}\right)$, $\operatorname{Rgn}\left(r_{a} q_{1} q_{2} r_{b}\right)$, and the new open region $\operatorname{Rgn}\left(r_{b} q_{2} p_{1} r_{3}\right)$ are convex sets. We consider two cases, and show that the theorem holds in each case.

Case 1: $\operatorname{Rgn}\left(r_{a} q_{1} q_{2} r_{b}\right)$ does not contain $p_{2}$ (see Fig. 5).

Proof. By Theorem A, the rooted tree $\left\langle B_{3} \cup\left\{w_{1}\right\}\right\rangle_{T_{1}}$ with root $w_{1}$ is strongly line embedded onto $\left(\operatorname{Rgn}\left(r_{a} q_{1} q_{2} r_{b}\right) \cap O(P)\right) \cup\left\{q_{1}\right\}$ with specified point $q_{1}$. It follows from Lemma 3 that the tree $\left\langle B_{2} \cup\left\{v_{1}, w_{1}\right\}\right\rangle_{T_{1}}$ with specified vertices $v_{1}$ and $w_{1}$ is strongly line embedded onto $\left(\operatorname{Rgn}\left(r_{a} q_{1} p_{1} r_{3}\right) \cap O(P)\right) \cup\left\{p_{1}, q_{1}\right\}$ with specified points $p_{1}$ and $q_{1}$. Moreover, by Lemma 5, the rooted forest $\left\langle B_{4}\right\rangle_{T_{1}} \cup\left\langle B_{1} \cup\left\{v_{1}\right\}\right\rangle_{T_{1}} \cup T_{2}$ with roots $w_{2}, v_{1}$, $v_{2}$ is strongly line embedded onto $\left(\operatorname{Rgn}\left(r_{b} q_{2} p_{1} r_{3}\right) \cap O(P)\right) \cup\left\{q_{2}, p_{1}, p_{2}\right\}$ with specified points $q_{2}, p_{1}$, and $p_{2}$. By adding the line segment $\overline{q_{1} q_{2}}$ to the above embeddings, we obtain a strong line embedding of $T_{1} \cup T_{2}$ onto $P$.

Case 2: $\operatorname{Rgn}\left(r_{a} q_{1} q_{2} r_{b}\right)$ contains $p_{2}$ (see Fig. 6).
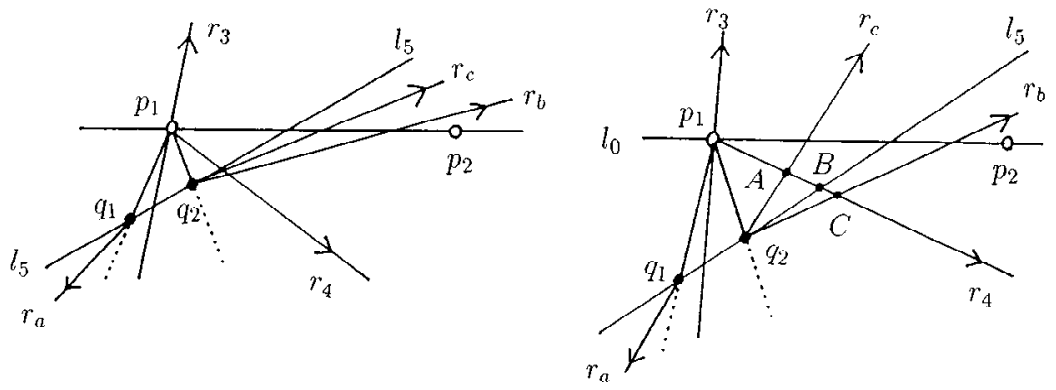

Fig. 6. $\operatorname{Rgn}\left(r_{a} q_{1} q_{2} r_{b}\right)$ contains $p_{2}$. 
Proof. We use Fig. 6. Since the open region $\operatorname{Rgn}\left(r_{b} q_{2} p_{1} r_{3}\right)$ contains $\left|B_{1}\right|+\left|B_{4}\right|-1+n_{2}$ ordinary points of $P$, we can take a ray $r_{c}$ emanating from $q_{2}$ and passing through no ordinary point of $P$ except $q_{2}$ such that the open region $\operatorname{Rgn}\left(r_{c} q_{2} p_{1} r_{3}\right)$ contains exactly $\left|B_{1}\right|+\left|B_{4}\right|-1$ ordinary points of $P$. In particular, the open region $\operatorname{Rgn}\left(r_{b} q_{2} r_{c}\right)$ contains exactly $n_{2}$ ordinary points of $P$.

If $r_{c}$ lies below $l_{5}$, then the rooted forest $\left\langle B_{1} \cup\left\{v_{1}\right\}\right\rangle_{T_{1}} \cup\left\langle B_{4}\right\rangle_{T_{1}}$ with roots $v_{1}$ and $w_{2}$ is strongly line embedded onto $\left(\operatorname{Rgn}\left(r_{c} q_{2} p_{1} r_{3}\right) \cap O(P)\right) \cup\left\{p_{1}, q_{2}\right\}$ with two specified points $p_{1}$ and $q_{2}$. Furthermore, by Lemma 4, the rooted forest $\left\langle B_{3} \cup\left\{w_{1}\right\}\right\rangle_{T_{1}} \cup T_{2}$ with root $w_{1}$ and $v_{2}$ is strongly line embedded onto $\left(\operatorname{Rgn}\left(r_{a} q_{1} q_{2} r_{c}\right) \cap O(P)\right) \cup\left\{q_{1}, p_{2}\right\}$ with two specified points $q_{1}$ and $p_{2}$. Hence, by adding the line segment $\overline{q_{1} q_{2}}$ to the above embeddings, we obtain a strong line embedding of $T_{1} \cup T_{2}$ onto $P$. Therefore we may assume that $r_{c}$ lies above $l_{5}$.

Let $A, B, C$ be the intersection points of $r_{4}$ and $r_{c}$, of $r_{4}$ and $l_{5}$, and of $r_{4}$ and $r_{b}$, respectively (see Fig. 6). Then, by the choice of $q_{1}$ and $q_{2}$, the open triangle $\triangle\left(p_{1} q_{2} B\right)$ contains no ordinary point of $P$. Moreover, it follows from the choice of $r_{4}$ and $r_{b}$ that the number of ordinary points of $P$ contained in the open triangle $\triangle\left(B q_{2} C\right)$ is equal to that of those points contained in the open region $\operatorname{Rgn}\left(r_{b} C r_{4}\right)$. Thus the open region $\operatorname{Rgn}\left(r_{c} A r_{4}\right)$ contains $n_{2}$ ordinary points. Then the rooted tree $T_{2}$ with root $v_{2}$ is strongly line embedded onto $\left(\operatorname{Rgn}\left(r_{4} A r_{c}\right) \cap O(P)\right) \cup\left\{p_{2}\right\}$ with specified point $p_{2}$.

By Lemma 4, the rooted forest $\left\langle B_{1} \cup\left\{v_{1}\right\}\right\rangle_{T_{1}} \cup\left\langle B_{4}\right\rangle_{T_{1}}$ with roots $v_{1}$ and $w_{2}$ is strongly line embedded onto $\left(\operatorname{Rgn}\left(r_{c} q_{2} p_{1} r_{3}\right) \cap O(P)\right) \cup\left\{p_{1}, q_{2}\right\}$ with two specified points $p_{1}$ and $q_{2}$. Moreover, since the open region $\operatorname{Rgn}\left(r_{a} q_{1} B r_{4}\right)$ contains $\left|B_{3}\right|$ ordinary points of $P$, the rooted tree $\left\langle B_{3} \cup\left\{w_{1}\right\}\right\rangle_{T_{1}}$ with root $w_{1}$ is strongly line embedded onto $\left(\operatorname{Rgn}\left(r_{a} q_{1} B r_{4}\right) \cap\right.$ $O(P)) \cup\left\{q_{1}\right\}$ with specified point $q_{1}$. By adding the line segment $\overline{q_{1} q_{2}}$ to the above embeddings, we obtain the desired strong line embedding of $T_{1} \cup T_{2}$ onto $P$.

Note that if $r_{c}$ intersects $r_{3}$ at a point $X$, then we consider the open triangle $\triangle\left(p_{1} q_{2} X\right)$ and the open region $\operatorname{Rgn}\left(r_{4} A r_{c} X r_{3}\right)$ instead of the open regions $\operatorname{Rgn}\left(r_{3} p_{1} q_{2} r_{c}\right)$ and $\operatorname{Rgn}\left(r_{4} A r_{c}\right)$, and then we obtain the desired strong line embedding of $T_{1} \cup T_{2}$.

Consequently the proof is complete.

We now show that the proof of Theorem 1 gives a $O\left(|F|^{2} \log |F|\right)$ time algorithm for finding a strong line embedding of $F=T_{1} \cup T_{2}$. We need the following theorem and lemma.

Theorem D [1]. Let $T$ be a rooted tree and let $P$ be a set of $|T|$ points in the plane in general position containing a specified point. Then we can strongly embed $T$ onto $P$ in $O(|T| \log |T|)$ time.

Theorem E (Theorem 3.7 of [6]). The convex hull of n points in the plane can be found in $O(n \log n)$ time.

We can find the vetex $v$ of a tree $T$ in Lemma 2 in $O(|T| \log |T|)$ time, and find a strong line embedding of a tree $T$ mentioned in Lemma 3 in $O\left(|T|^{2} \log |T|\right)$ time by making use of Theorems E and D. By similar arguments, we can show that a strong line embedding of a rooted forest $F$ mentioned in Lemma 4 and one of a rooted forest $F$ 
mentioned in Lemma 5 are found in $O\left(|F|^{2} \log |F|\right)$ time each. Therefore we can say that the required strong line embedding of a rooted forest $F$ consisting of two rooted trees is also found in $O\left(|F|^{2} \log |F|\right)$ time by the above results and by the proof of the theorem.

\section{Acknowledgments}

The authors would like to thank Hitoshi Suzuki for his helpful suggestions on the complexities of algorithms. They would also like to thank the referees for their helpful comments.

\section{References}

1. P. Bose, M. McAllister, and J. Snoeyink, Optimal algorithms to embed trees in a point set, Graph Drawing (Proceeding of Symposium on Graph Drawing, DG '95), Lecture Notes in Computer Sciences, Vol. 1027 (1996), Springer-Verlag, Berlin, pp. 64-75.

2. H. de Fraysseix, P. Pach, and R. Pollack, How to draw a planar graph on a grid, Combinatorica 10 (1990), 41-51.

3. Y. Ikeba, M. Perles, A. Tamura, and S. Tokunaga, The rooted tree embedding problem into points on the plane, Discrete Comput. Geom. 11 (1994), 51-63.

4. J. Pach and J. Törőcsik, Layout of rooted tree, Planar Graphs, DIMACS Series in Discrete Mathematics and Theoretical Computer Science, Vol. 9 (1993), pp. 131-137.

5. M. Perles, Open problem proposed at the DIMACS Workshop on Arrangements, Rutgers University, 1990.

6. F. Preparata and M. Shamos, Computational Geometry, Springer-Verlag (1985).

7. S.Tokunaga, On a straight-line embedding problem of graphs, Discrete Math. 150 (1996), 371-378.

Received July 3, 1997, and in revised form February 25, 1998. 